\title{
THE BURGESS INEQUALITY AND THE LEAST $k$-TH POWER NON-RESIDUE
}

\author{
ENRIQUE TREVIÑO
}

\begin{abstract}
The Burgess inequality is the best upper bound we have for the character sum $S_{\chi}(M, N)=\sum_{M<n \leq M+N} \chi(n)$. Until recently, no explicit estimates had been given for the inequality. In 2006, Booker gave an explicit estimate for quadratic characters which he used to calculate the class number of a 32-digit discriminant. McGown used an explicit estimate to show that there are no norm-Euclidean Galois cubic fields with discriminant greater than $10^{140}$. Both of their explicit estimates are on restricted ranges. In this paper we prove an explicit estimate that works for any $M$ and $N$. We also improve McGown's estimates in a slightly narrower range, getting explicit estimates for characters of any order. We apply the estimates to the question of how large must a prime $p$ be to ensure that there is a $k$-th power non-residue less than $p^{1 / 6}$.
\end{abstract}

\section{INTRODUCTION}

Let $\chi$ be a character $\bmod q$ for some integer $q>1$. Throughout the paper we will use the term character for Dirichlet character. Let $S_{\chi}(M, N)$ be defined as follows

$$
S_{\chi}(M, N)=\sum_{M<n \leq M+N} \chi(n) .
$$

Historically, studying this sum has proven fruitful in analytic number theory to bound the least $k$-th power non-residue, to bound class numbers, to bound the least inert prime in a number field, and to bound the least primitive root, among other applications. The first non-trivial bound for this sum was proven independently by Pólya and Vinogradov in 1918; namely, they showed that there exists an absolute constant $c>0$ such that $\left|S_{\chi}(M, N)\right| \leq c \sqrt{q} \log q$. The Pólya-Vinogradov inequality is very useful when $N$ is big compared to $\sqrt{q}$, but not very useful otherwise (since trivially $\left.\left|S_{\chi}(M, N)\right| \leq N\right)$. What we want is an inequality that gives a nontrivial result even when $N$ is small compared to $\sqrt{q}$. The best theorem for short character sums is known as the Burgess inequality ([5], [6], [8], [9]) and allows us to take $N$ as small as $q^{\frac{1}{4}-o(1)}$ 目 We state the theorem below:

Theorem (Burgess). Let $\chi$ be a primitive character $\bmod q$ with $q>1$, and let $M$ and $N$ be non-negative reals with $N \geq 1$. Then

$$
\left|S_{\chi}(M, N)\right| \ll N^{1-\frac{1}{r}} q^{\frac{r+1}{4 r^{2}}+\varepsilon}
$$

2010 Mathematics Subject Classification. Primary 11L40, 11Y60.

Key words and phrases. Character Sums, Burgess inequality, power residues and non-residues. Many results in this paper are in Chapter 6 of the author's Ph. D. Dissertation [20].

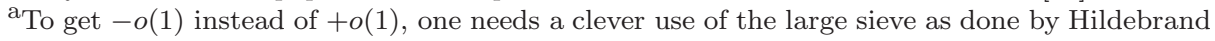
in 13 . 
for $r=2,3$ and for any $r \geq 1$ if $q$ is cubefree, the implied constant depending only on $\varepsilon$ and $r$.

To prove the Burgess inequality, one of the keys is the following inequality which relies on a deep theorem of Weil [23]:

Theorem A. For $p$ a prime number, $r$ a positive integer and $B$ a positive real number satisfying $r \leq 9 B$, let $\chi$ be a non-principal character to the modulus $p$. Then

$$
\sum_{x \bmod p}\left|\sum_{1 \leq b \leq B} \chi(x+b)\right|^{2 r} \leq(2 r-1) ! ! B^{r} p+(2 r-1) B^{2 r} \sqrt{p} .
$$

where $(2 r-1) ! !=(2 r-1)(2 r-3) \ldots(1)$.

The above theorem was proven with weaker constants by Erdös and Davenport in [10, and Burgess improved it to better constants and used it to get the Burgess inequality. In [3], Booker proved it with these constants for quadratic characters. In [21, the author extended it to all characters. The reliance on the Weil estimate makes it difficult to improve the Burgess inequality asymptotically.

Recently, some problems have required getting explicit estimates on the Burgess inequality. Booker in [3] needed an explicit form of the inequality to compute a 32digit discriminant. McGown in [15] used an explicit form of the inequality to show that there are no norm-Euclidean Galois cubic fields of discriminant greater than $10^{140}$. The goal of this paper is to improve their explicit estimates in the ranges they work in and give an explicit estimate that works regardless of the range of $N$. We apply these estimates to a question about $k$-th power non-residues $\bmod p$.

The work of Booker and McGown relies on the exposition of the Burgess inequality in 14. In that book, Iwaniec and Kowalski sketch the proof of the following:

Theorem B. Let $p$ be a large enough prime. Let $\chi$ be a non-principal character $\bmod p$. Let $r$ be a positive integer, and let $M$ and $N$ be non-negative integers with $N \geq 1$. Then

$$
\left|S_{\chi}(M, N)\right| \leq 30 N^{1-\frac{1}{r}} p^{\frac{r+1}{4 r^{2}}}(\log p)^{\frac{1}{r}} .
$$

In Section 3 we improve Theorem B to

Theorem 1. Let $p$ be a prime. Let $\chi$ be a non-principal character $\bmod p$. Let $M$ and $N$ be non-negative integers with $N \geq 1$, let $2 \leq r \leq 10$ be a positive integer, and let $p_{0}$ be a positive real number. Then for $p \geq p_{0}$, there exists $c_{1}(r)$, a constant depending on $r$ and $p_{0}$ such that

$$
\left|S_{\chi}(M, N)\right| \leq c_{1}(r) N^{1-\frac{1}{r}} p^{\frac{r+1}{4 r^{2}}}(\log p)^{\frac{1}{r}},
$$

where $c_{1}(r)$ is given by Table 1

In the spirit of Theorem $\mathrm{B}$, where we have no restriction on $r$, we also prove the following corollary:

Corollary 1. Let $p$ be a prime such that $p \geq 10^{7}$. Let $\chi$ be a non-principal character $\bmod p$. Let $r$ be a positive integer, and let $M$ and $N$ be non-negative integers with $N \geq 1$. Then

$$
\left|S_{\chi}(M, N)\right| \leq 2.74 N^{1-\frac{1}{r}} p^{\frac{r+1}{4 r^{2}}}(\log p)^{\frac{1}{r}} .
$$




\begin{tabular}{|c|c|c|c|}
\hline$r$ & $p_{0}=10^{7}$ & $p_{0}=10^{10}$ & $p_{0}=10^{20}$ \\
\hline 2 & 2.7381 & 2.5173 & 2.3549 \\
\hline 3 & 2.0197 & 1.7385 & 1.3695 \\
\hline 4 & 1.7308 & 1.5151 & 1.3104 \\
\hline 5 & 1.6107 & 1.4572 & 1.2987 \\
\hline 6 & 1.5482 & 1.4274 & 1.2901 \\
\hline 7 & 1.5052 & 1.4042 & 1.2813 \\
\hline 8 & 1.4703 & 1.3846 & 1.2729 \\
\hline 9 & 1.4411 & 1.3662 & 1.2641 \\
\hline 10 & 1.4160 & 1.3495 & 1.2562 \\
\hline
\end{tabular}

TABLE 1. Values for the constant $c_{1}(r)$ in the Burgess inequality.

Restricting $N$ to be less than $4 p^{\frac{1}{2}+\frac{1}{4 r}}$, McGown in 15 proved an explicit version of Burgess with worse constants but with a better exponent in $\log p$. Indeed, he proved:

Theorem C. Let $p \geq 2 \cdot 10^{4}$ be a prime number. Let $M$ and $N$ be non-negative integers with $1 \leq N \leq 4 p^{\frac{1}{2}+\frac{1}{4 r}}$. Suppose $\chi$ is a non-principal character $\bmod p$. Then there exists a computable constant $C(r)$ such that

$$
\left|S_{\chi}(M, N)\right|<C(r) N^{1-\frac{1}{r}} p^{\frac{r+1}{4 r^{2}}}(\log p)^{\frac{1}{2 r}},
$$

where $C(r)$ is given by Table 0

\begin{tabular}{|c|c|c|c|}
\hline$r$ & $C(r)$ & $r$ & $C(r)$ \\
\hline 2 & 10.0366 & 9 & 2.1467 \\
\hline 3 & 4.9539 & 10 & 2.0492 \\
\hline 4 & 3.6493 & 11 & 1.9712 \\
\hline 5 & 3.0356 & 12 & 1.9073 \\
\hline 6 & 2.6765 & 13 & 1.8540 \\
\hline 7 & 2.4400 & 14 & 1.8088 \\
\hline 8 & 2.2721 & 15 & 1.7700 \\
\hline
\end{tabular}

TABLE 2. Values for the constant $C(r)$ in the Burgess inequality.

The restriction that $N \leq 4 p^{\frac{1}{2}+\frac{1}{4 r}}$ is used to get the exponent $\frac{1}{2 r}$ in the $\log p$ term of the inequality. In Section 4 , we improve McGown's Theorem to have better constants in a similar range.

Theorem 2. Let $p$ be a prime. Let $\chi$ be a non-principal character $\bmod p$. Let $M$ and $N$ be non-negative integers with $1 \leq N \leq 2 p^{\frac{1}{2}+\frac{1}{4 r}}$, let $r \leq 10$ be a positive integer, and let $p_{0}$ be a positive real number. Then for $p \geq p_{0}$, there exists $c_{2}(r)$, a constant depending on $r$ and $p_{0}$ such that

$$
\left|S_{\chi}(M, N)\right|<c_{2}(r) N^{1-\frac{1}{r}} p^{\frac{r+1}{4 r^{2}}}(\log p)^{\frac{1}{2 r}}
$$

where $c_{2}(r)$ is given by Table 3 .

Using an idea from [16, we can get rid of the restriction on $N$ for $r \geq 3$. 


\begin{tabular}{|c|c|c|c|}
\hline$r$ & $p_{0}=10^{10}$ & $p_{0}=10^{15}$ & $p_{0}=10^{20}$ \\
\hline 2 & 3.6529 & 3.5851 & 3.5751 \\
\hline 3 & 2.5888 & 2.5144 & 2.4945 \\
\hline 4 & 2.1914 & 2.1258 & 2.1078 \\
\hline 5 & 1.9841 & 1.9231 & 1.9043 \\
\hline 6 & 1.8508 & 1.7959 & 1.7757 \\
\hline 7 & 1.7586 & 1.7066 & 1.6854 \\
\hline 8 & 1.6869 & 1.6384 & 1.6187 \\
\hline 9 & 1.6283 & 1.5857 & 1.5654 \\
\hline 10 & 1.5794 & 1.5410 & 1.5216 \\
\hline
\end{tabular}

TABLE 3. Values for the constant $c_{2}(r)$ in the Burgess inequality.

Corollary 2. Let $p \geq 10^{10}$ be a prime number. Let $M$ and $N$ be non-negative integers with $N \geq 1$. Suppose $\chi$ is a non-principal character $\bmod p$ and that $p \geq p_{0}$ for some positive real $p_{0}$. Then for $r \geq 3$, there exists a computable constant $c_{2}(r)$ depending on $r$ and $p_{0}$, such that

$$
\left|S_{\chi}(M, N)\right|<c_{2}(r) N^{1-\frac{1}{r}} p^{\frac{r+1}{4 r^{2}}}(\log p)^{\frac{1}{2 r}},
$$

where $c_{2}(r)$ is the same as that of Table ? whenever $r \geq 3$.

Putting an extra restriction on $N$ (namely, $N \leq 2 \sqrt{p}$ ), Booker in [3] gave better bounds in the special case of quadratic characters.

Remark 1. Using Theorem A one could extend Booker's theorem to all orders of $\chi$ (with slightly worse constants). The reason we would get slightly worse constants is that in the quadratic case, the inequality in Theorem A can be improved slightly to $(2 r-1) ! ! B^{r} p+(2 r-2) B^{2 r} \sqrt{p}$. Every other part of Booker's proof extends naturally, but this part of the inequality fails when looking at higher orders.

In Section 5 we apply these estimates to a question about $k$-th power nonresidues $\bmod p$. Indeed, let $p$ be a prime and let $k$ be an integer with $k \mid p-1$ and $k>1$. Let $g(p, k)$ be the least $k$-th power non-residue $\bmod p$. The case $k=2$, i.e., the question of how big the least quadratic non-residue is, has been studied extensively. A probabilistic heuristic using that a prime $q$ is a quadratic non-residue $\bmod p$ half of the time, suggests that $g(p, 2) \ll \log p \log \log p$ and that $g(p, 2) \gg$ $\log p \log \log p$ for infinitely many $p$. Assuming the Generalized Riemann Hypothesis for Dirichlet L-functions (GRH), Ankeny [1] showed that $g(p, k) \ll(\log p)^{2}$ and Bach 2] made this explicit by proving (under GRH) that $g(p, k) \leq 2(\log p)^{2}$. The best unconditional results (for $g(p, k)$ ) are due to Burgess [5], who, building on work by Vinogradov [22], showed that

$$
g(p, k) \ll_{\varepsilon} p^{\frac{1}{4 \sqrt{e}}+\varepsilon} .
$$

For $k \geq 3$, better estimates which depend upon $k$ have been proven by Wang Yuan([24]) building on work of Vinogradov ([22]) and Buhštab (4

All of the work described so far has been of asymptotic nature. In terms of getting explicit bounds, Karl Norton [18, building on a technique of Burgess [7, was able to show that $g(p, k) \leq 3.9 p^{1 / 4} \log p$ unless $k=2$ and $p \equiv 3(\bmod 4)$ for which he showed $g(p, k) \leq 4.7 p^{1 / 4} \log p$. In 21], the author improved Norton's 
bounds to $0.9 p^{1 / 4} \log p$ and $1.1 p^{1 / 4} \log p$, respectively. These bounds are far from the asymptotic bound of $p^{\frac{1}{4 \sqrt{e}}+\varepsilon}$. In this paper, as an application of the explicit Burgess inequality, we find an upper bound on how large $p$ has to be to ensure that there is a $k$-th power non-residue less than $p^{1 / 6}$.

Theorem 3. Let $p$ be a prime number and $k>1$ be a positive divisor of $p-1$. Then for $p \geq 10^{4732}$, the least $k$-th power non-residue $\bmod p$ is less than or equal to $p^{1 / 6}$.

Remark 2. The techniques involved in the proof can be used to answer this question for $p^{\alpha}$ whenever $\alpha>\frac{1}{4 \sqrt{e}}$.

\section{Preliminary Lemmas}

Let $A$ and $N$ be positive integers. Let $v(x)$ be the number of representations of $x$ as $\bar{a} n(\bmod p)$, where $\bar{a}$ is the inverse of $a(\bmod p), 1 \leq a \leq A$ and $M<n \leq M+N$, that is,

(1) $v(x)=\#\left\{(a, n) \in \mathbb{N}^{2} \mid 1 \leq a \leq A, M<n \leq M+N\right.$ and $\left.n \equiv a x \bmod p\right\}$.

The main lemma in this section is the following:

Lemma 1. Let $p$ be a prime and let $N<p$ be a positive integer. Let $A \geq 28$ be an integer satisfying $A<\frac{N}{12}$, then

$$
V_{2}=\sum_{x \bmod p} v^{2}(x) \leq 2 A N\left(\frac{A N}{p}+\log (1.85 A)\right) .
$$

To prove the lemma regarding $V_{2}$ we will need a couple of estimates involving the $\phi$ function (Lemmas 2 and 3), an estimate on a sum of logarithms (Lemma 4) and a non-trivial combinatorial count (Lemma 5).

Lemma 2. For $x \geq 1$ a real number we have:

$$
\sum_{n \leq x} \frac{\phi(n)}{n} \leq \frac{6}{\pi^{2}} x+\log x+1
$$

Proof. For $1 \leq x<2$, the left hand side of (3) is 1 , while the right hand side is at least 1 . We can manually check that for all integers $x$ satisfying $2 \leq x \leq 41$ we have

$$
\sum_{n \leq x} \frac{\phi(n)}{n} \leq \frac{6}{\pi^{2}}(x-1)+\log (x-1)+1,
$$

implying that (3) is true for $x<41$. Therefore, we may assume that $x \geq 41$.

Let's work with the sum:

$$
\sum_{n \leq x} \frac{\phi(n)}{n}=\sum_{n \leq x} \frac{1}{n} \sum_{d \mid n} \mu(d) \frac{n}{d}=\sum_{d \leq x} \sum_{n \leq \frac{x}{d}} \frac{\mu(d)}{d}=\sum_{d \leq x}\left\lfloor\frac{x}{d}\right\rfloor \frac{\mu(d)}{d} .
$$

From [12, Theorem 422] it follows that for $x \geq 1$

$$
\sum_{d \leq x} \frac{1}{d}<\log x+\gamma+\frac{1}{x} .
$$


Using (5) in (4) yields

(6) $\sum_{n \leq x} \frac{\phi(n)}{n} \leq x \sum_{d>1} \frac{\mu(d)}{d^{2}}-x \sum_{d>x} \frac{\mu(d)}{d^{2}}+\sum_{d \leq x} \frac{1}{d} \leq \frac{6}{\pi^{2}} x+\log x+\gamma+\frac{1}{x}-x \sum_{d>x} \frac{\mu(d)}{d^{2}}$.

Moser and Macleod 17] gave a simple proof that for $x \geq 2$ we have

$$
\left|\sum_{d>x} \frac{\mu(d)}{d^{2}}\right| \leq \frac{1}{3 x}+\frac{8}{3 x^{2}} \text {. }
$$

Combining (7) with (6) yields for $x \geq 41$ that

$$
\sum_{n \leq x} \frac{\phi(n)}{n} \leq \frac{6}{\pi^{2}} x+\log x+\gamma+\frac{1}{x}+\frac{1}{3}+\frac{8}{3 x} \leq \frac{6}{\pi^{2}} x+\log x+1 .
$$

Lemma 3. For $x \geq 1$ a real number we have:

$$
\sum_{n \leq x} n \phi(n) \leq \frac{2}{\pi^{2}} x^{3}+\frac{1}{2} x^{2} \log x+x^{2} .
$$

Proof. For $1 \leq x<2$, the left hand side of (8) is 1 , while the right hand side is at least $x^{2} \geq 1$. Therefore it is true for $1 \leq x<2$. Now for $2 \leq x<3$, the left hand side is 3 , while the right hand side is at least $x^{2} \geq 4$. Therefore (8) is true for $1 \leq x<3$. In the rest of the proof we will assume that $x \geq 3$. Let's work with the sum:

$$
\begin{aligned}
\sum_{n \leq x} \phi(n) n=\sum_{n \leq x} \sum_{d \mid n} \frac{\mu(d) n^{2}}{d}=\sum_{d \leq x} & \mu(d) d \sum_{d m \leq x} m^{2} \\
& =\sum_{d \leq x} \frac{\mu(d) d}{6}\left\lfloor\frac{x}{d}\right\rfloor\left(\left\lfloor\frac{x}{d}\right\rfloor+1\right)\left(2\left\lfloor\frac{x}{d}\right\rfloor+1\right) .
\end{aligned}
$$

Now, let $\theta_{d}=\frac{x}{d}-\left\lfloor\frac{x}{d}\right\rfloor$. Then we have

$$
\begin{aligned}
\sum_{n \leq x} \phi(n) n= & \frac{x^{3}}{3} \sum_{d \leq x} \frac{\mu(d)}{d^{2}}+\frac{x^{2}}{6} \sum_{d \leq x} \frac{\left(3-6 \theta_{d}\right) \mu(d)}{d} \\
& +\frac{x}{6} \sum_{d \leq x}\left(6 \theta_{d}^{2}-6 \theta_{d}+1\right) \mu(d)-\frac{1}{6} \sum_{d \leq x} \theta_{d}\left(1-\theta_{d}\right)\left(1-2 \theta_{d}\right) \mu(d) d .
\end{aligned}
$$

From (5) it follows that for $x \geq 3$

$$
\sum_{d \leq x} \frac{1}{d}<\log x+\gamma+\frac{1}{x}<\log x+1-\frac{1}{60}-\frac{1}{60 x} .
$$

Using that $0 \leq \theta_{d} \leq 1$ we have that $\left|3-6 \theta_{d}\right| \leq 3$, that $\left|6 \theta_{d}^{2}-6 \theta_{d}+1\right| \leq 1$ and $\left|\left(1-\theta_{d}\right)\left(1-2 \theta_{d}\right)\left(-\theta_{d}\right)\right| \leq \frac{1}{10}$. Therefore, using (9), (10), that $\sum_{d \geq 1} \frac{\mu(d)}{d^{2}}=\frac{6}{\pi^{2}}$, and that $|\mu(d)| \leq 1$, we get

$$
\sum_{n \leq x} \phi(n) n \leq \frac{x^{3}}{3} \sum_{d \leq x} \frac{\mu(d)}{d^{2}}+\frac{x^{2}}{2} \sum_{d \leq x} \frac{1}{d}+\frac{x}{6} \sum_{d \leq x} 1+\frac{1}{60} \sum_{d \leq x} d
$$


$\leq \frac{2}{\pi^{2}} x^{3}-\frac{x^{3}}{3} \sum_{d>x} \frac{\mu(d)}{d^{2}}+\frac{1}{2} x^{2} \log x+\frac{x^{2}}{2}-\frac{x^{2}}{120}-\frac{x}{120}+\frac{x^{2}}{6}+\frac{1}{60}\left(\frac{x(x+1)}{2}\right)$.

From (7) we have (for $x \geq 2$ )

$$
\sum_{d>x} \frac{\mu(d)}{d^{2}} \geq \frac{1}{3 x}-\frac{8}{3 x^{2}} \geq-\frac{1}{x}
$$

Combining this with (11) yields the lemma.

Lemma 4. For $x \geq 1$ we have:

$$
\sum_{d \leq x} \log \left(\frac{x}{d}\right) \leq x-1
$$

Proof. For $1 \leq x<2$ we have $\sum_{d \leq x} \log \left(\frac{x}{d}\right)=\log x \leq x-1$. Therefore, we may assume $x \geq 2$. Now,

$$
\sum_{d \leq x} \log \left(\frac{x}{d}\right)=\lfloor x\rfloor \log x-\sum_{d \leq x} \log d \leq\lfloor x\rfloor \log x-\lfloor x\rfloor \log \lfloor x\rfloor+\lfloor x\rfloor-1 .
$$

To get the second inequality we used that

$$
\sum_{d \leq x} \log d=\sum_{2 \leq d \leq x} \log d \geq \int_{1}^{\lfloor x\rfloor} \log t d t=\lfloor x\rfloor \log \lfloor x\rfloor-\lfloor x\rfloor+1 .
$$

Now, notice that $x=\lfloor x\rfloor+\{x\}$ and $\log (1+y) \leq y$. Therefore we have

$$
\lfloor x\rfloor \log x=\lfloor x\rfloor \log \lfloor x\rfloor+\lfloor x\rfloor \log (x /\lfloor x\rfloor) \leq\lfloor x\rfloor \log \lfloor x\rfloor+\{x\} .
$$

Combining equations (12) and (13) yields

$$
\sum_{d \leq x} \log \left(\frac{x}{d}\right) \leq\{x\}+\lfloor x\rfloor-1=x-1 .
$$

Lemma 5. Let $A \geq 2, N \geq 1, a_{1}, a_{2}$ and $M$ be integers. Let $p>N$ be a prime number. Suppose, $1 \leq a_{1}, a_{2} \leq A$ with $a_{1} \neq a_{2}$. Then the number of pairs of integers $\left(n_{1}, n_{2}\right)$ satisfying $M<n_{1}, n_{2} \leq N+M$ and $a_{1} n_{2}-a_{2} n_{1}=k p$ is bounded above by

$$
N \frac{\operatorname{gcd}\left(a_{1}, a_{2}\right)}{\max \left\{a_{1}, a_{2}\right\}}+1
$$

Proof. Let $d=\operatorname{gcd}\left(a_{1}, a_{2}\right)$. Since $a_{1} n_{2}-a_{2} n_{1}=k p$, we have that $d \mid k$. Let $a_{1}=a_{1}^{\prime} d, a_{2}=a_{2}^{\prime} d$ and $k=k^{\prime} d$. Now, we also have

$$
n_{2}=\frac{k p+a_{2} n_{1}}{a_{1}}=\frac{k^{\prime} p+a_{2}^{\prime} n_{1}}{a_{1}^{\prime}} .
$$

The right hand side of (14) must be an integer. Therefore $k^{\prime} p+a_{2}^{\prime} n_{1} \equiv 0 \bmod a_{1}^{\prime}$. Since this is a linear equation in terms of $n_{1}$, there is at most one solution mod $a_{1}^{\prime}$. Therefore, in the interval $(M, M+N]$ there are at most $\frac{N}{a_{1}^{\prime}}+1$ choices of $n_{1}$. 
Since $n_{2}$ is uniquely determined from $n_{1}$, the number of pairs $\left(n_{1}, n_{2}\right)$ satisfying the conditions of the lemma is bounded by

$$
\frac{N}{a_{1}^{\prime}}+1=N \frac{\operatorname{gcd}\left(a_{1}, a_{2}\right)}{a_{1}}+1
$$

Analogously, the number of pairs is bounded by $N \frac{\operatorname{gcd}\left(a_{1}, a_{2}\right)}{a_{2}}+1$. The statement of the lemma is now an easy consequence.

Now we are ready to prove Lemma 1

Proof of Lemma 1. We'll begin by noting that $V_{2}$ is the number of quadruples $\left(a_{1}, a_{2}, n_{1}, n_{2}\right)$ with $1 \leq a_{1}, a_{2} \leq A$ and $M<n_{1}, n_{2} \leq M+N$ such that $a_{1} n_{2} \equiv a_{2} n_{1}$ $(\bmod p)$. If $a_{1}=a_{2}$, since $N<p$, we have that $n_{1}=n_{2}$ because $n_{1} \equiv n_{2}(\bmod p)$ while $\left|n_{1}-n_{2}\right| \leq N<p$. Therefore, the number of quadruples in this case is $A N$. Fix $a_{1}$ and $a_{2}$ with $a_{1} \neq a_{2}$. Let $k$ be an integer satisfying

$$
a_{1} n_{2}-a_{2} n_{1}=k p,
$$

for some $n_{1}$ and $n_{2}$. We can put a bound on possible values for $k$. First of all, $k$ must be a multiple of $\operatorname{gcd}\left(a_{1}, a_{2}\right)$. Now, if we write $n_{1}=n_{1}^{\prime}+M$ and $n_{2}=n_{2}^{\prime}+M$, we have, using $k p-\left(a_{1}-a_{2}\right) M=a_{1} n_{2}^{\prime}-a_{2} n_{1}^{\prime}$, that

$$
-a_{2} N \leq-a_{2} n_{1}^{\prime}<k p-\left(a_{1}-a_{2}\right) M<a_{1} n_{2}^{\prime} \leq a_{1} N .
$$

Therefore $k$ lies in an interval of length at most $\frac{\left(a_{1}+a_{2}\right) N}{p}$. Since $k$ is a multiple of $\operatorname{gcd}\left(a_{1}, a_{2}\right)$ and $k$ lies in such an interval, then there are at most

$$
\frac{\left(a_{1}+a_{2}\right) N}{\operatorname{gcd}\left(a_{1}, a_{2}\right) p}+1
$$

choices for $k$.

Given $a_{1}, a_{2}$ and $k$ we can count the number of pairs $\left(n_{1}, n_{2}\right)$ which would satisfy (15). By Lemma 5 the number of pairs is bounded by $N \frac{\operatorname{gcd}\left(a_{1}, a_{2}\right)}{\max \left\{a_{1}, a_{2}\right\}}+1$. Therefore we get

$$
\begin{array}{r}
V_{2} \leq A N+2 \sum_{a_{1}<a_{2}}\left(\frac{\left(a_{1}+a_{2}\right) N}{\operatorname{gcd}\left(a_{1}, a_{2}\right) p}+1\right)\left(\frac{\operatorname{gcd}\left(a_{1}, a_{2}\right) N}{\max \left\{a_{1}, a_{2}\right\}}+1\right) \\
=A N+\frac{2 N^{2}}{p} S_{1}+\frac{2 N}{p} S_{2}+2 N S_{3}+A^{2}-A,
\end{array}
$$

where

$$
\begin{aligned}
& S_{1}=\sum_{a_{1}<a_{2}} \frac{a_{1}+a_{2}}{\max \left\{a_{1}, a_{2}\right\}}, \\
& S_{2}=\sum_{a_{1}<a_{2}} \frac{a_{1}+a_{2}}{\operatorname{gcd}\left(a_{1}, a_{2}\right)},
\end{aligned}
$$

and

$$
S_{3}=\sum_{a_{1}<a_{2}} \frac{\operatorname{gcd}\left(a_{1}, a_{2}\right)}{\max \left\{a_{1}, a_{2}\right\}}
$$


Dealing with $S_{1}$ is straightforward, in fact $S_{1}$ is (18)

$$
\sum_{a_{2} \leq A} \sum_{a_{1}<a_{2}} \frac{a_{1}+a_{2}}{a_{2}}=\sum_{a_{2} \leq A}\left(a_{2}-1+\frac{a_{2}\left(a_{2}-1\right)}{2 a_{2}}\right)=\frac{3}{2} \frac{A(A-1)}{2}=\frac{3}{4} A^{2}-\frac{3}{4} A .
$$

Now, let's estimate $S_{2}$ :

$$
\begin{aligned}
S_{2}=\sum_{a_{1}<a_{2} \leq A} \frac{a_{1}+a_{2}}{\operatorname{gcd}\left(a_{1}, a_{2}\right)}=\sum_{d \leq A} \sum_{b_{2} \leq \frac{A}{d}} \sum_{b_{1}<b_{2},\left(b_{1}, b_{2}\right)=1}\left(b_{1}+b_{2}\right) \\
=\sum_{d \leq A} \sum_{2 \leq b_{2} \leq \frac{A}{d}}\left(\frac{\phi\left(b_{2}\right)}{2} b_{2}+\phi\left(b_{2}\right) b_{2}\right)=\frac{3}{2} \sum_{d \leq A} \sum_{2 \leq b_{2} \leq \frac{A}{d}} \phi\left(b_{2}\right) b_{2} .
\end{aligned}
$$

Using Lemma 3, we get

$$
S_{2} \leq \frac{3}{\pi^{2}} \sum_{d \leq A}\left(\frac{A}{d}\right)^{3}+\frac{3}{4} \sum_{d \leq A}\left(\frac{A}{d}\right)^{2} \log \left(\frac{A}{d}\right)+\frac{3}{2} \sum_{d \leq A}\left(\frac{A}{d}\right)^{2} .
$$

Using that $\log \left(\frac{A}{d}\right)=\log A-\log d$, and that $\sum_{d \geq 1} \frac{1}{d^{s}}=\zeta(s)$, yields

$$
S_{2} \leq \frac{3 \zeta(3)}{\pi^{2}} A^{3}+\frac{3 \zeta(2)}{4} A^{2} \log A-\frac{3}{4} A^{2} \sum_{d \leq A} \frac{\log d}{d^{2}}+\frac{3}{2} A^{2} \zeta(2) .
$$

Using that for $A \geq 11$ we have $\frac{3 \zeta(2)}{2}-\frac{3}{4} \sum_{d \leq A} \frac{\log d}{d^{2}}<2$ yields

$$
S_{2} \leq \frac{3 \zeta(3)}{\pi^{2}} A^{3}+\frac{3 \zeta(2)}{4} A^{2} \log (A)+2 A^{2} .
$$

Let's estimate $S_{3}$. We have

$$
S_{3}=\sum_{a_{1}<a_{2} \leq A} \frac{\operatorname{gcd}\left(a_{1}, a_{2}\right)}{\max \left(a_{1}, a_{2}\right)}=\sum_{d \leq A} \sum_{b_{2} \leq \frac{A}{d}} \sum_{b_{1}<b_{2},\left(b_{1}, b_{2}\right)=1} \frac{1}{b_{2}}=\sum_{d \leq A} \sum_{2 \leq b_{2} \leq \frac{A}{d}} \frac{\phi\left(b_{2}\right)}{b_{2}} .
$$

Using Lemma 2 yields

$$
S_{3} \leq \sum_{d \leq A}\left(\frac{A}{d} \frac{1}{\zeta(2)}+\log \left(\frac{A}{d}\right)\right)=\frac{6}{\pi^{2}} A \sum_{d \leq A} \frac{1}{d}+\sum_{d \leq A} \log \left(\frac{A}{d}\right) .
$$

From (5) it follows that for $A \geq 27$

$$
\sum_{d \leq A} \frac{1}{d}<\log A+\gamma+\frac{1}{A}<\log (1.85 A) .
$$

Using this and Lemma 4 yields

$$
S_{3} \leq \frac{6}{\pi^{2}} A \log (1.85 A)+A-1 .
$$

Using (18), (19) and (20) in (16) yields the following upper bound for $V_{2}$ :

$2 A N\left(\frac{3}{2}+\frac{A-1}{2 N}+\frac{3 A N}{4 p}-\frac{3 N}{4 p}+\frac{3 \zeta(3) A^{2}}{\pi^{2} p}+\frac{3 \zeta(2) A \log A}{4 p}+\frac{6}{\pi^{2}} \log (1.85 A)-\frac{1}{A}+\frac{2 A}{p}\right)$

For $A \geq 4$, we have

$$
\frac{3 \zeta(3) A^{2}}{\pi^{2} p}+\frac{3 \zeta(2) A \log A}{4 p}<\frac{3}{4} \frac{A^{2}}{p}
$$


Since $N>3 A$ we have the following two inequalities:

$$
\frac{A N}{4 p}>\frac{3}{4} \frac{A^{2}}{p} \text { and } \frac{3 N}{4 p}>\frac{2 A}{p} .
$$

Combining (22) and (23) yields

$$
\frac{3 A N}{4 p}+\left(\frac{3 \zeta(3) A^{2}}{\pi^{2} p}+\frac{3 \zeta(2) A \log A}{4 p}\right)+\left(\frac{2 A}{p}-\frac{3 N}{4 p}\right)<\frac{A N}{p} .
$$

Finally, using that $A \geq 28$ and that $N>12 A$, yields

(25) $\left(1-\frac{6}{\pi^{2}}\right) \log (1.85 A) \geq\left(1-\frac{6}{\pi^{2}}\right) \log (51.8) \geq 1.54766>\frac{3}{2}+\frac{1}{24} \geq \frac{3}{2}+\frac{A}{2 N}$.

Combining (24) and (25) in (21) yields (2).

Remark 3. The main term will come from the $\log (1.85 A)$ term and the 1.85 can be changed to a smaller number (the limit being $e^{\gamma}$ ), forcing $A$ to be slightly larger to make the inequalities work. Also, the coefficient on $\log (1.85 A)$ can be changed to be as close to $\frac{6}{\pi^{2}}$ as we want as long as $A$ is big enough. It is important to note that big $A$ 's will mean forcing $p$ to be much bigger in the estimates for the Burgess inequality.

Remark 4. The constraint $A \geq 28$ is used to get the main term to be $\log (1.85 A)$; however, we can relax the condition on $A$ and get a slightly worse main term. We chose our values this way to get the constants in Tables 1 and 3 as low as possible for small values of $r$. Relaxing the $A \geq 28$ condition would make these constants worse, but improve the constants for larger values of $r$. Since the small values of $r$ seem to be the most useful in applications, we decided to focus on minimizing these cases.

\section{Explicit Burgess inequality}

Proof of Theorem 1 Let $M$ and $N \geq 1$ be non-negative integers. Let $r \geq 2$ be a positive integer. Fix a constant $c_{1}(r) \geq 1$ (which we will name later). We will prove the Theorem by induction. Assume that for all positive integers $h<N$, we have

$$
\left|S_{\chi}(M, h)\right| \leq c_{1}(r) h^{1-\frac{1}{r}} p^{\frac{r+1}{4 r^{2}}}(\log p)^{\frac{1}{r}} .
$$

The idea is to estimate $S_{\chi}(M, N)$ by shifting by $h(n \mapsto n+h)$ and getting an error that we can deal with by induction.

Note that, for all $h<N$,

$$
S_{\chi}(M, N)=\sum_{M<n \leq M+N} \chi(n+h)+\sum_{M<n \leq M+h} \chi(n)-\sum_{M+N<n \leq M+N+h} \chi(n) .
$$

Therefore

$$
S_{\chi}(M, N)=\sum_{M<n \leq N+M} \chi(n+h)+2 \theta E(h),
$$

where $|\theta| \leq 1$ which depends upon $h$, and $E(h)=\max _{K}\left|S_{\chi}(K, h)\right|$.

Let $A$ and $B$ be positive reals and let $H=\lfloor A\rfloor\lfloor B\rfloor$. We will use shifts of length $h=a b$ where $a$ and $b$ are positive integers satisfying $a \leq A$ and $b \leq B$. After averaging over all the pairs $(a, b)$ we get

$$
S_{\chi}(M, N)=\frac{1}{H} \sum_{a, b} \sum_{M<n \leq M+N} \chi(n+a b)+\frac{1}{H} \sum_{a, b} 2 \theta E(a b) .
$$


Let $v(x)$ be defined as in (10), then

$$
\left|\sum_{a, b} \sum_{M<n \leq M+N} \chi(n+a b)\right| \leq \sum_{x} v(x)\left|\sum_{b \leq B} \chi(x+b)\right| .
$$

Let

$$
V:=\sum_{x} v(x)\left|\sum_{b \leq B} \chi(x+b)\right| .
$$

Then, combining (26) with (27), we get

$$
\left|S_{\chi}(M, N)\right| \leq \frac{V}{H}+\frac{2}{H} \sum_{a, b} E(a b)
$$

We can now focus on estimating $V$. Now define $V_{1}:=\sum_{x(\bmod p)} v(x)$,

$V_{2}:=\sum_{x(\bmod p)} v^{2}(x)$ and $W:=\sum_{x(\bmod p)}\left|\sum_{1 \leq b \leq B} \chi(x+b)\right|^{2 r}$. Using Hölder's Inequality we get

$$
V \leq V_{1}^{1-\frac{1}{r}} V_{2}^{\frac{1}{2 r}} W^{\frac{1}{2 r}}
$$

First note that

$$
V_{1}=\lfloor A\rfloor N \leq A N .
$$

From Lemma 1] for $\lfloor A\rfloor \geq 28$ and $\lfloor A\rfloor<\frac{N}{12}$, we have

$$
V_{2} \leq 2 A N\left(\frac{A N}{p}+\log (1.85 A)\right) .
$$

We can also bound $W$, since by Theorem A, we have (for $r \leq 9 B$ ):

$$
W \leq \frac{(2 r) !}{2^{r} r !} B^{r} p+(2 r-1) B^{2 r} \sqrt{p}=(2 r-1) ! ! B^{r} p+(2 r-1) B^{2 r} \sqrt{p} .
$$

Let's head back to proving the Burgess bound. We will let $A B=k N$ for $k$ a real number to be chosen later. Using the inequalities of $V_{1}, V_{2}$ and $W$ together with (29) yields the following bound upper bound for $\frac{V}{H}$ :

$$
\begin{gathered}
\frac{V}{H} \leq \frac{1}{\lfloor A\rfloor\lfloor B\rfloor} V_{1}^{1-\frac{1}{r}} V_{2}^{\frac{1}{2 r}} W^{\frac{1}{2 r}} \leq \frac{\frac{A B}{\lfloor A\rfloor[B\rfloor}}{(A B)^{\frac{1}{2 r}}} \cdot \frac{(2 W B)^{\frac{1}{2 r}}}{B}\left(\frac{A N}{p}+\log (1.85 A)\right)^{\frac{1}{2 r}} N^{1-\frac{1}{2 r}} \\
\leq \frac{A}{A-1} \cdot \frac{B}{B-1} \cdot \frac{1}{k^{\frac{1}{2 r}}} \cdot \frac{(2 W B)^{\frac{1}{2 r}}}{B}\left(\frac{A N}{p}+\log (1.85 A)\right)^{\frac{1}{2 r}} N^{1-\frac{1}{r}}
\end{gathered}
$$

Because of (32) we can see that a good choice for $B$ is the one that minimizes $\frac{W B}{B^{2 r}}$. Using (31), we seek to minimize the expression $(2 r-1) ! ! \frac{p}{B^{r-1}}+(2 r-1) B p^{\frac{1}{2}}$. We take the derivative with respect to $B$ and equal it to zero. After this process we get that a $\operatorname{good} B$ is

$$
B=((2 r-3) ! !(r-1))^{\frac{1}{r}} p^{\frac{1}{2 r}} .
$$


Using this value of $B$ we get

$$
\frac{(2 W B)^{\frac{1}{2 r}}}{B} \leq\left(\frac{2 r(2 r-1)}{r-1}\right)^{\frac{1}{2 r}}(r-1)^{\frac{1}{2 r^{2}}}((2 r-3) ! !)^{\frac{1}{2 r^{2}}} p^{\frac{r+1}{4 r^{2}}} .
$$

Now we must try to bound $\frac{A N}{p}+\log (1.85 A)$. To do this, we can use the PólyaVinogradov inequality to give an upper bound for $N$, since for $N$ large, the PólyaVinogradov inequality would be a better bound than the Burgess inequality. Indeed, if

$$
N \geq p^{\frac{1}{2}+\frac{1}{4 r}} \log p
$$

then, since $c_{1}(r) \geq 1$, we have

$$
c_{1}(r) N^{1-\frac{1}{r}} p^{\frac{r+1}{4 r^{2}}}(\log p)^{\frac{1}{r}} \geq \sqrt{p} \log p .
$$

Therefore, from the Pólya-Vinogradov inequality (see Section 9.4 in [16]) we can conclude that $\left|S_{\chi}(M, N)\right| \leq c_{1}(r) N^{1-\frac{1}{r}} p^{\frac{r+1}{4 r^{2}}}(\log p)^{\frac{1}{r}}$, whenever we have (35).

If we have $r \geq 3$, then we can use the Burgess inequality with $r-1$ instead of the Pólya-Vinogradov inequality, to get a better upper bound on $N$. Indeed, if we let $s$ be a real number that satisfies

$$
c_{1}(r-1) \leq s^{\frac{1}{r(r-1)}} c_{1}(r),
$$

then if

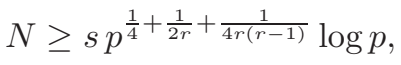

then

$$
c_{1}(r) N^{1-\frac{1}{r}} p^{\frac{r+1}{4 r^{2}}}(\log p)^{\frac{1}{r}} \geq c_{1}(r-1) N^{1-\frac{1}{r-1}} p^{\frac{r}{4(r-1)^{2}}}(\log p)^{\frac{1}{r-1}} .
$$

Similarly, we can put a lower bound on $N$, by noting that $\left|S_{\chi}(M, N)\right| \leq N$. Indeed,

$$
c_{1}(r) N^{1-\frac{1}{r}} p^{\frac{r+1}{4 r^{2}}}(\log p)^{\frac{1}{r}} \geq N
$$

whenever

$$
N \leq c_{1}(r)^{r} p^{\frac{1}{4}+\frac{1}{4 r}} \log p .
$$

Therefore, we may assume that

$$
c_{1}(2)^{2} p^{\frac{3}{8}} \log p<N<p^{\frac{5}{8}} \log p
$$

for $r=2$, and that

$$
c_{1}(r)^{r} p^{\frac{1}{4}+\frac{1}{4 r}} \log p<N<s p^{\frac{1}{4}+\frac{1}{2 r}+\frac{1}{4 r(r-1)} \log p,}
$$

for $r \geq 3$.

Using that $A=\frac{k N}{B}$, the upper bound for $N$ in (37), and (33), we get

$$
\frac{A N}{p}=\frac{k N^{2}}{p B} \leq \frac{k p^{\frac{5}{4}} \log ^{2} p}{p B} \leq k \log ^{2} p
$$

for $r=2$, and for $r \geq 3$, we get

$$
\frac{A N}{p}=\frac{k N^{2}}{p B} \leq \frac{s^{2} k p^{\frac{1}{2}+\frac{1}{r}+\frac{1}{2 r(r-1)} \log ^{2} p}}{p B}=\frac{s^{2} k}{((2 r-3) ! !(r-1))^{\frac{1}{r}} p^{\frac{1}{2}-\frac{1}{2 r}-\frac{1}{2 r(r-1)}}} \log ^{2} p,
$$

Now we consider what happens to $\log (1.85 A)$.

$$
\log (1.85 A)=\log \left(\frac{1.85 k N}{B}\right) \leq \log (1.85 k \log p)+\frac{3 \log p}{8},
$$


for $r=2$, and for $r \geq 3$, we get

(42)

$$
\log (1.85 A)=\log \left(\frac{1.85 k N}{B}\right) \leq \log \left(\frac{1.85 s k \log p}{((2 r-3) ! !(r-1))^{\frac{1}{r}}}\right)+\frac{\log p}{4}+\frac{\log p}{4 r(r-1)}
$$

Now, let's bound the error term, the part we have labeled as $E(h)$.

For any $a, b$ such that $a b=h<N$, we have by induction hypothesis $E(h) \leq$ $c_{1}(r)(a b)^{1-\frac{1}{r}} p^{\frac{r+1}{4 r^{2}}}(\log p)^{\frac{1}{r}}$. Therefore,

$$
\begin{gathered}
\frac{1}{c_{1}(r) p^{\frac{r+1}{4 r^{2}}}(\log p)^{\frac{1}{r}}} \cdot \frac{2}{H} \sum_{a, b} E(a b) \leq \frac{2}{\lfloor A\rfloor\lfloor B\rfloor} \sum_{1 \leq a \leq A} \sum_{1 \leq b \leq B}(a b)^{1-\frac{1}{r}} \\
\leq 2 \frac{1}{A B}\left(\int_{1}^{A+1} t^{1-\frac{1}{r}} d t\right)\left(\int_{1}^{B+1} t^{1-\frac{1}{r}} d t\right) \frac{A B}{(A-1)(B-1)} \\
\leq(A B)^{1-\frac{1}{r}} \frac{2}{\left(2-\frac{1}{r}\right)^{2}}\left(\frac{(A+1)(B+1)}{A B}\right)^{2-\frac{1}{r}} \frac{A B}{(A-1)(B-1)} \\
=\frac{2 r^{2}}{(2 r-1)^{2}} k^{1-\frac{1}{r}} N^{1-\frac{1}{r}}\left(\frac{(A+1)(B+1)}{A B}\right)^{2-\frac{1}{r}} \frac{A B}{(A-1)(B-1)} .
\end{gathered}
$$

Combining equations (32), (34), (39), (41) and (43) with (28) yields (for $r=2$ )

$$
\begin{array}{r}
\frac{\left|S_{\chi}(M, N)\right|}{N^{\frac{1}{2}} p^{\frac{3}{16}}(\log p)^{\frac{1}{2}}} \leq \frac{A B}{(A-1)(B-1)}(12)^{\frac{1}{4}}\left(1+\frac{3}{8 k \log p}+\frac{\log (1.85 k \log p)}{k \log ^{2} p}\right)^{\frac{1}{4}} \\
+\frac{8}{9} k^{\frac{1}{2}} c_{1}(2)\left(\frac{(A+1)(B+1)}{A B}\right)^{\frac{3}{2}} \frac{A B}{(A-1)(B-1)} .
\end{array}
$$

Similarly, for $r \geq 3$, combining equations (32), (34), (40), (42) and (43) with (28) yields

$$
\frac{\left|S_{\chi}(M, N)\right|}{N^{1-\frac{1}{r}} p^{\frac{r+1}{4 r^{2}}}(\log p)^{\frac{1}{r}}} \leq\left(\frac{2 r(2 r-1)}{r-1}\right)^{\frac{1}{2 r}}((2 r-3) ! !(r-1))^{\frac{1}{2 r^{2}}} \frac{A B}{(A-1)(B-1)}
$$

$$
\begin{gathered}
\left(\frac{s^{2}}{((2 r-3) ! !(r-1))^{\frac{1}{r}} p^{\frac{r-2}{2(r-1)}}}+\frac{1}{4 k \log p}+\frac{1}{4 r(r-1) k \log p}+\frac{\log \left(\frac{1.85 s k \log p}{((2 r-3) ! !(r-1))^{\frac{1}{r}}}\right)}{k \log ^{2} p}\right)^{\frac{1}{2 r}} \\
+\frac{2 r^{2}}{(2 r-1)^{2}} k^{1-\frac{1}{r}} c_{1}(r)\left(\frac{(A+1)(B+1)}{A B}\right)^{2-\frac{1}{r}} \frac{A B}{(A-1)(B-1)} .
\end{gathered}
$$

Now, if we let $c_{1}(r)$ be defined as follows

$$
c_{1}(2)=\frac{A B}{(A-1)(B-1)}(12)^{\frac{1}{4}} \frac{\left(1+\frac{3}{8 k \log p}+\frac{\log (1.85 k \log p)}{k \log ^{2} p}\right)^{\frac{1}{4}}}{1-\frac{8}{9} k^{\frac{1}{2}}\left(\frac{(A+1)(B+1)}{A B}\right)^{\frac{3}{2}}\left(\frac{A B}{(A-1)(B-1)}\right)},
$$

for $r=2$, and

$$
c_{1}(r)=\frac{A B}{(A-1)(B-1)}\left(\frac{2 r(2 r-1)((2 r-3) ! !(r-1))^{\frac{1}{r}}}{r-1}\right)^{\frac{1}{2 r}}
$$




$$
\frac{\left(\frac{s^{2}}{((2 r-3) ! !(r-1))^{\frac{1}{r}} p^{\frac{1}{2}-\frac{1}{2 r}-\frac{1}{2 r(r-1)}}}+\frac{1}{4 k \log p}+\frac{1}{4 r(r-1) k \log p}+\frac{\log \left(\frac{1.85 s k \log p}{((2 r-3) !(r-1))^{\frac{1}{r}}}\right)}{k \log ^{2} p}\right)^{\frac{1}{2 r}}}{1-\frac{2 r^{2}}{(2 r-1)^{2}} k^{1-\frac{1}{r}}\left(\frac{(A+1)(B+1)}{A B}\right)^{2-\frac{1}{r}}\left(\frac{A B}{(A-1)(B-1)}\right)},
$$

for $r \geq 3$. Therefore from (44) and (45), we get that

$$
\left|S_{\chi}(M, N)\right| \leq c_{1}(r) N^{1-\frac{1}{r}} p^{\frac{r+1}{4 r^{2}}}(\log p)^{\frac{1}{r}} .
$$

All we have to do is pick $k$ to minimize $c_{1}(r)$ in such a way that $\lfloor A\rfloor \geq 28$, and that $N \geq 12 A$. First, we'll start by showing that

$$
B \geq 15 \text {. }
$$

Since $B=((2 r-3) ! !(r-1))^{\frac{1}{r}} p^{\frac{1}{2 r}}$, we can just manually check for $2 \leq r \leq 20$ that the inequality is satisfied. To show that it works for $r \geq 21$, we can show that

$$
((2 r-3) ! !(r-1))^{\frac{1}{r}} \geq 15,
$$

by noticing that it works for $r=21$ and that the left hand side of (48) is increasing. Indeed, the left hand side is increasing; by noticing that $(2 r-3)(r-1)<(2 r-$ 1) $(r+1)$, we get

$$
(2 r-3) ! !(r-1)<\frac{(2 r-1)^{r-1}(r+1)^{r-1}}{(r-1)^{r-2}}<\frac{(2 r-1)^{r}(r+1)^{r}}{(r-1)^{r}},
$$

implying that

$$
\frac{1}{r} \log ((2 r-3) ! !(r-1))<\log ((2 r-1)(r+1))-\log (r-1),
$$

which implies

$$
\frac{r+1}{r} \log ((2 r-3) ! !(r-1))<\log ((2 r-1) ! !)+\log (r+1),
$$

and hence

$$
\left.\log \left(((2 r-3) ! !(r-1))^{\frac{1}{r}}\right)<\log (((2 r-1) ! !)(r+1))^{\frac{1}{r+1}}\right) .
$$

Using that $B \geq 15$, since $A=\frac{k N}{B}$, then

$$
A=\frac{k N}{B}<\frac{k N}{12}<\frac{N}{12}
$$

whenever $k<1$.

Let $k \geq \frac{1}{30}$. To check that $\lfloor A\rfloor \geq 28$, we use (38) and (33) and we note that

$$
\lfloor A\rfloor \geq A-1 \geq \frac{N}{30 B}-1 \geq \frac{c_{1}(r)^{r} p^{\frac{1}{4}-\frac{1}{4 r}} \log p}{30((2 r-3) ! !(r-1))^{\frac{1}{r}}}-1 .
$$

Table 4 shows the lower bound $c_{1}(r)$ must satisfy to have $\lfloor A\rfloor \geq 28$ in different situations.

Now, $B$ is defined in terms of $r$ and $p$. By fixing an $r$ and a $p_{0}$ (a fixed lower bound for $p$ ), we can calculate $B$ in terms of $r$ and $p_{0}$. Let $c_{1}^{\prime}(r)$ be a parameter satisfying $c_{1}^{\prime}(r)<c_{1}(r) . A$ is written in terms of $k$ and $N$ and from (37) and (38) we have a range for $N$ in terms of $c_{1}(r), p, r, k$ and $s$. From this we can find a lower bound for $A$ in terms of $c_{1}^{\prime}(r), k, r, s$ and $p$. The parameter $s$ is optimal when it is as small as possible so we fix $s$ (in terms of $r, c_{1}^{\prime}(r)$ and $c_{1}(r-1)$ ) to 


\begin{tabular}{|c|c|c|c|}
\hline$r$ & $p \geq 10^{7}$ & $p \geq 10^{10}$ & $p \geq 10^{20}$ \\
\hline 2 & 2.68289 & 1.45765 & 0.24442 \\
\hline 3 & 1.88354 & 1.13939 & 0.251637 \\
\hline 4 & 1.6153 & 1.06881 & 0.305418 \\
\hline 5 & 1.48379 & 1.04807 & 0.363232 \\
\hline 6 & 1.40512 & 1.04167 & 0.417191 \\
\hline 7 & 1.35216 & 1.04007 & 0.465518 \\
\hline 8 & 1.31369 & 1.04016 & 0.508197 \\
\hline 9 & 1.28422 & 1.04077 & 0.545749 \\
\hline 10 & 1.26077 & 1.04147 & 0.578819 \\
\hline
\end{tabular}

TABLE 4. Lower bounds for the constant $c_{1}(r)$ in the Burgess inequality to satisfy $\lfloor A\rfloor \geq 28$.

be the smallest real satisfying (36). After plugging in $A, B, r, s, k, p_{0}$ and $c_{1}^{\prime}(r)$ to the equations (46) and (47), we can find a good value of $k \in\left[\frac{1}{30}, 1\right)$, and a good value of $c_{1}^{\prime}(r)$ for each $r$ and $p_{0}$ to find the smallest $c_{1}(r)$ we can. After making the choices of $k$ and $c_{1}^{\prime}(r)$ described in Table 5 , we conclude that $c_{1}(r)$ has the values listed in Table 1 as upper bounds.

\begin{tabular}{|c|c|c|c|c|c|c|}
\cline { 2 - 7 } \multicolumn{1}{c|}{} & \multicolumn{2}{c|}{$p_{0}=10^{7}$} & \multicolumn{2}{c|}{$p_{0}=10^{10}$} & \multicolumn{2}{c|}{$p_{0}=10^{20}$} \\
\hline$r$ & $k$ & $c_{1}^{\prime}(r)$ & $k$ & $c_{1}^{\prime}(r)$ & $k$ & $c_{1}^{\prime}(r)$ \\
\hline 2 & $2 / 45$ & 2.738 & $1 / 30$ & 2.517 & $1 / 30$ & 2.354 \\
\hline 3 & $1 / 16$ & 2.019 & $11 / 150$ & 1.737 & $2 / 15$ & 1.369 \\
\hline 4 & $1 / 12$ & 1.729 & $31 / 300$ & 1.515 & $37 / 300$ & 1.310 \\
\hline 5 & $1 / 12$ & 1.610 & $7 / 75$ & 1.456 & $31 / 300$ & 1.298 \\
\hline 6 & $1 / 12$ & 1.548 & $1 / 12$ & 1.426 & $7 / 75$ & 1.289 \\
\hline 7 & $11 / 150$ & 1.504 & $11 / 150$ & 1.404 & $1 / 12$ & 1.281 \\
\hline 8 & $19 / 300$ & 1.470 & $19 / 300$ & 1.383 & $1 / 12$ & 1.272 \\
\hline 9 & $19 / 300$ & 1.441 & $19 / 300$ & 1.366 & $11 / 150$ & 1.264 \\
\hline 10 & $4 / 75$ & 1.415 & $4 / 75$ & 1.349 & $11 / 150$ & 1.256 \\
\hline
\end{tabular}

TABLE 5. Values chosen for $k$ and $c_{1}^{\prime}(r)$ to build Table 1 .

Proof of Corollary 1. We begin by pointing out that Theorem 1 proves this for $2 \leq r \leq 10$ and $p \geq 10^{7}$. We also know that it is true for the $r=1$ case by the Pólya-Vinogradov inequality (Vinogradov proved it with the constant 1 in [22]).

Following the proof of Theorem 1 we also have that $B \geq 15$ for all $r$ and hence, for any $k<1$, we have $A<\frac{N}{12}$. It is also worth pointing out that we can use $s=1$, since now the constant 2.74 is fixed as the constant in our upper bound, instead of a constant depending on $r$.

We need to show that you can pick a $k$ such that $\lfloor A\rfloor \geq 28$. First, let's prove that $2.74^{r} \geq((2 r-3) ! !(r-1))^{\frac{1}{r}}$. Indeed, for all $r \geq 1$ we have

$$
2.74^{r}>2 r \geq((2 r-3) ! !(r-1))^{\frac{1}{r}} \text {. }
$$


Now we have

$$
A=\frac{k N}{B} \geq \frac{k(2.74)^{r} p^{\frac{1}{4}-\frac{1}{4 r}} \log p}{((2 r-3) ! !(r-1))^{\frac{1}{r}}} \geq k p^{\frac{1}{4}-\frac{1}{4 r}} \log p>29,
$$

whenever $k>\frac{29}{p^{\frac{1}{4}-\frac{1}{4 r}} \log p}$.

We replace $B$ by 15 in (47) (since $B \geq 15$ ), and we can see that the only factors that don't decrease with $r$ are the $k^{1-\frac{1}{r}}$ term which appears in the denominator, and the $\left(2-\frac{1}{r}\right)$ exponent in the denominator. With this in mind, let $c(r)$ be defined as follows for $r \geq 4$ :

$$
c(r)=\frac{15 A}{14(A-1)}\left(\frac{2 r(2 r-1)((2 r-3) ! !(r-1))^{\frac{1}{r}}}{r-1}\right)^{\frac{1}{2 r}}
$$

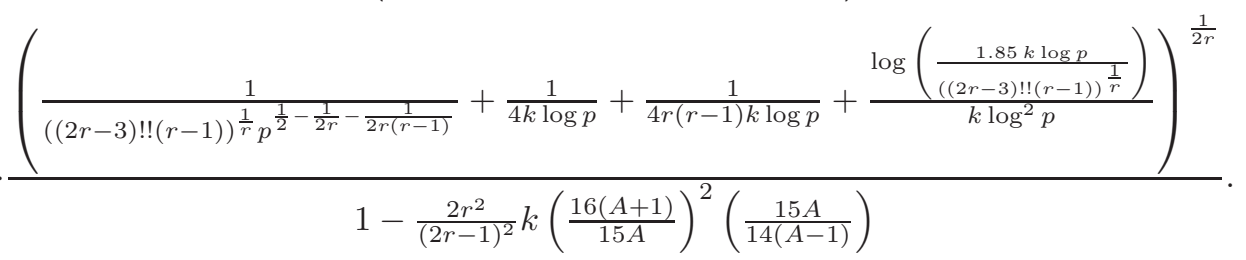

Letting $k=\frac{11}{64}, A \geq k p^{\frac{1}{4}-\frac{1}{4 r}}$ and $p \geq 10^{7}$ we confirm that $c(r) \leq 2.74$ whenever $r \geq 3$. Since it is also true for $r \leq 3$, we conclude our corollary.

\section{IMPROVING MCGOWN'S THEOREM}

The main obstacle in improving the $(\log p)^{\frac{1}{r}}$ factor in the Burgess inequality is the bound on $V_{2}$. However, if we put a bound on $N$, we can make the proof cleaner while also improving the exponent in $\log p$ to $\frac{1}{2 r}$. First we prove a lemma regarding $V_{2}$ and then we will be able to prove Theorem 2

Lemma 6. Let $p$ be a prime, and $N$ be a positive integer. Let $A \geq 30$ be an integer such that $N>7 A$ and $2 A N<p$. Let $v(x)$ be defined as in (11). Then

$$
V_{2}=\sum_{x} v^{2}(x) \leq 2 A N \log (1.85 A) .
$$

Proof. The proof is essentially the same as that of Lemma 1. Recall that $V_{2}$ is the number of quadruples $\left(a_{1}, a_{2}, n_{1}, n_{2}\right)$ with $1 \leq a_{1}, a_{2} \leq A$ and $M<n_{1}, n_{2} \leq M+N$ such that $a_{1} n_{2} \equiv a_{2} n_{1}(\bmod p)$. If $a_{1}=a_{2}$, since $N<p$, we have that $n_{1}=n_{2}$ because $n_{1} \equiv n_{2}(\bmod p)$ while $\left|n_{1}-n_{2}\right| \leq N<p$. Therefore, the number of quadruples in this case is $A N$. Fixing $a_{1} \neq a_{2}$ and writing

$$
a_{1} n_{2}-a_{2} n_{1}=k p,
$$

we can put a bound on possible values for $k$. As shown in the proof of Lemma 1 , there are at most $\frac{\left(a_{1}+a_{2}\right) N}{\operatorname{gcd}\left(a_{1}, a_{2}\right) p}+1$ values of $k$. Since $2 A N<p$, then we have that $k$ is uniquely determined.

In the proof of Lemma 1, we showed that given $a_{1}, a_{2}$ and $k$, the number of pairs $\left(n_{1}, n_{2}\right)$ is bounded by $N \frac{\operatorname{gcd}\left(a_{1}, a_{2}\right)}{\max \left\{a_{1}, a_{2}\right\}}+1$.

Now, for $A \geq 30$ and $N>7 A$ we have

$$
\left(1-\frac{6}{\pi^{2}}\right) \log (1.85 A) \geq\left(1-\frac{6}{\pi^{2}}\right) \log (55.5)=1.57471>\frac{3}{2}+\frac{1}{14}>\frac{3}{2}+\frac{A}{2 N}
$$


Using the definition of $S_{3}$ as in (17), using the inequalities (20) and (50), for $A \geq 30$ and $N>7 A$, we have

$$
\begin{aligned}
V_{2} \leq A N+2 \sum_{a_{1}<a_{2}}\left(\frac{\operatorname{gcd}\left(a_{1}, a_{2}\right) N}{\max \left\{a_{1}, a_{2}\right\}}+1\right) \\
\quad=A N+2 N S_{3}+A^{2}-A \leq 2 A N \log (1.85 A) .
\end{aligned}
$$

Now we are ready to prove Theorem 2

Proof of Theorem [. The proof is very similar to the proof of Theorem1 We proceed by induction, assuming that for all $h<N$ we have $\left|S_{\chi}(M, h)\right| \leq c_{2}(r) p^{\frac{r+1}{4 r^{2}}}(\log p)^{\frac{1}{2 r}}$.

Most of the work in the proof of Theorem 1 can be replicated. So I'll just point out the things that change.

The first change is that by employing Lemma 6. (30) becomes

$$
V_{2} \leq 2 A N \log (1.85 A) \text {. }
$$

This change affects (32), by deleting $\frac{A N}{p}$ inside the parenthesis. Now it looks as follows:

$$
\frac{V}{H} \leq \frac{A B}{(A-1)(B-1)} \frac{1}{k^{\frac{1}{2 r}}} \frac{(2 W B)^{\frac{1}{2 r}}}{B}(\log (1.85 A))^{\frac{1}{2 r}} N^{1-\frac{1}{r}} .
$$

The next change is the range for $N$, which we deduced by using the PólyaVinogradov inequality, the trivial bound, and the case for $r-1$. Instead of (37), using our hypothesis and the trivial bound, we now have

$$
c_{2}(2)^{r} p^{\frac{3}{8}} \sqrt{\log p}<N<2 p^{\frac{5}{8}},
$$

for $r=2$. Assuming $c_{2}(r-1) \leq s^{\frac{1}{r(r-1)}} c_{2}(r)$ for a real number $s$, and using the Burgess inequality for $r-1$ we have, for $r \geq 3$, the following range for $N$

$$
c_{2}(r)^{r} p^{\frac{1}{4}+\frac{1}{4 r}} \sqrt{\log p}<N<\min \left\{2 p^{\frac{1}{2}+\frac{1}{4 r}}, s p^{\frac{1}{4}+\frac{1}{2 r}+\frac{1}{4 r(r-1)}} \sqrt{\log p}\right\} .
$$

Using that $A=\frac{k N}{B}$ and (52), we get

$$
\log (1.85 A)=\log \left(\frac{1.85 k N}{B}\right) \leq \log (3.7 k)+\frac{3 \log p}{8} \leq \frac{3 \log p}{8},
$$

for $r=2$ (we're assuming $k<1 / 4$, which implies $\log (3.7 k)<0$ ). Using (153), yields

$$
\log (1.85 A)=\log \left(\frac{1.85 k N}{B}\right) \leq \log \left(\frac{1.85 s k \sqrt{\log p}}{((2 r-3) ! !(r-1))^{\frac{1}{r}}}\right)+\frac{\log p}{4}+\frac{\log p}{4 r(r-1)},
$$

for $r \geq 3$.

The bound for $E(h)$ is almost the same as in (43), the only difference being the exponent of $\log p$, which is now $\frac{1}{2 r}$ instead of $\frac{1}{r}$. Making this change and using both (34) and (54) with (51) yields (for $r=2$ )

$$
\begin{aligned}
& \frac{\left|S_{\chi}(M, N)\right|}{N^{\frac{1}{2}} p^{\frac{3}{16}}(\log p)^{\frac{1}{4}}} \leq \frac{A B}{(A-1)(B-1)}(12)^{\frac{1}{4}}\left(\frac{3}{8 k}\right)^{\frac{1}{4}} \\
& +\frac{A B}{(A-1)(B-1)} \frac{8}{9} k^{\frac{1}{2}} c_{2}(2)\left(\frac{(A+1)(B+1)}{A B}\right)^{\frac{3}{2}}
\end{aligned}
$$


For $r \geq 3$, using (34) and (55) with (51) yields

$$
\begin{gathered}
\frac{\left|S_{\chi}(M, N)\right|}{N^{1-\frac{1}{r}} p^{\frac{r+1}{4 r^{2}}}(\log p)^{\frac{1}{2 r}}} \leq \frac{A B}{(A-1)(B-1)}\left(\frac{2 r(2 r-1)((2 r-3) ! !(r-1))^{\frac{1}{r}}}{r-1}\right)^{\frac{1}{2 r}} \\
\quad\left(\frac{\log \left(\frac{1.85 s k \sqrt{\log p}}{((2 r-3) ! !(r-1))^{\frac{1}{r}}}\right)}{k \log p}+\frac{1}{4 k}+\frac{1}{4 r(r-1) k}\right)^{\frac{1}{2 r}} \\
+\frac{A B}{(A-1)(B-1)} \frac{2 r^{2}}{(2 r-1)^{2}} k^{1-\frac{1}{r}} c_{2}(r)\left(\frac{(A+1)(B+1)}{A B}\right)^{2-\frac{1}{r}}
\end{gathered}
$$

Now, if we let $c_{2}(r)$ be defined as follows

$$
c_{2}(2)=\frac{A}{A-1} \frac{B}{B-1} \frac{(12)^{\frac{1}{4}}\left(\frac{3}{8 k}\right)^{\frac{1}{4}}}{1-\frac{8}{9} k^{\frac{1}{2}}\left(\frac{(A+1)(B+1)}{A B}\right)^{\frac{3}{2}}\left(\frac{A B}{(A-1)(B-1)}\right)},
$$

and, for $r \geq 3$,

$$
c_{2}(r)=\frac{A}{A-1} \frac{B}{B-1} \frac{\left(\frac{2 r(2 r-1)\left((2 r-3) ! !(r-1) \frac{1}{r}\right.}{r-1}\left(\frac{\log \left(\frac{1.85 s k \sqrt{\operatorname{og} p}}{\left((2 r-3) ! !(r-1) \frac{1}{r}\right.}\right)}{k \log p}+\frac{1}{4 k}+\frac{1}{4 r(r-1) k}\right)\right)^{\frac{1}{2 r}}}{1-\frac{2 r^{2}}{(2 r-1)^{2}} k^{1-\frac{1}{r}}\left(\frac{(A+1)(B+1)}{A B}\right)^{2-\frac{1}{r}}\left(\frac{A B}{(A-1)(B-1)}\right)},
$$

for $r \geq 3$. Then, from (56) and (57), we get that

$$
\left|S_{\chi}(M, N)\right| \leq c_{2}(r) N^{1-\frac{1}{r}} p^{\frac{r+1}{4 r^{2}}}(\log p)^{\frac{1}{2 r}} .
$$

All we have to do is pick $k$ to minimize $c_{2}(r)$ in such a way that $\lfloor A\rfloor \geq 30$, that $N>7 A$ and $2 A N<p$.

Using that $B \geq 15$, it is not hard to check that $N \geq 7 A$. Indeed, since $A=\frac{k N}{B}$, we have $A \leq \frac{k N}{15}<\frac{N}{7}$.

To check that $\lfloor A\rfloor \geq 30$ for $k \geq \frac{3}{64}$, we do the following:

$$
\lfloor A\rfloor \geq A-1 \geq \frac{3 N}{64 B}-1 \geq \frac{3 c_{2}(r)^{r} p^{\frac{1}{4}-\frac{1}{4 r}} \sqrt{\log p}}{64((2 r-3) ! !(r-1))^{\frac{1}{r}}}-1 .
$$

Table 6 shows the lower bound $c$ must satisfy to have $\lfloor A\rfloor \geq 30$ in different situations.

Let's now verify that $2 A N<p$. Indeed, from the fact that $A=\frac{k N}{B}$ and from (52), we have

$$
2 A N=\frac{2 k N^{2}}{B} \leq \frac{8 k p}{((2 r-3) ! !(r-1))^{\frac{1}{r}}}<p,
$$

whenever $k<\min \left\{\frac{((2 r-3) ! !(r-1))^{\frac{1}{r}}}{8}, 1\right\}$.

As in the proof of Theorem 1, we define can find bounds for $c_{2}(r)$ by controlling the parameters $p_{0}, c_{2}^{\prime}(r)$ and $k$. We find a good value of $k \in\left[\frac{3}{64}, \frac{((2 r-3) ! !(r-1))^{\frac{1}{r}}}{8}\right)$ and a good value of $c_{2}^{\prime}(r)$ for each $r$ and $p_{0}$, and plug in the values of $B, k$, and a 


\begin{tabular}{|c|c|c|c|}
\hline$r$ & $p \geq 10^{10}$ & $p \geq 10^{15}$ & $p \geq 10^{20}$ \\
\hline 2 & 2.78392 & 1.22500 & 0.55514 \\
\hline 3 & 1.75393 & 0.86474 & 0.43480 \\
\hline 4 & 1.47708 & 0.81850 & 0.46029 \\
\hline 5 & 1.35767 & 0.82260 & 0.50431 \\
\hline 6 & 1.29240 & 0.83775 & 0.54839 \\
\hline 7 & 1.25127 & 0.85450 & 0.58848 \\
\hline 8 & 1.22279 & 0.87022 & 0.62388 \\
\hline 9 & 1.20171 & 0.88422 & 0.65489 \\
\hline 10 & 1.18536 & 0.89649 & 0.68202 \\
\hline
\end{tabular}

TABLE 6. Lower bounds for the constant $c_{2}(r)$ in the Burgess inequality to satisfy $\lfloor A\rfloor \geq 30$.

lower bound bound for $A$ on (58) to find $c_{2}(2)$ and on (59) to find $c_{2}(r)$ for $r \geq 3$ in Table 3 and conclude the theorem. The values of $k$ and $c_{2}^{\prime}(r)$ we chose can be found on Table 7

\begin{tabular}{|c|c|c|c|c|c|c|}
\cline { 2 - 7 } \multicolumn{1}{c|}{} & \multicolumn{2}{c|}{$p_{0}=10^{10}$} & \multicolumn{2}{c|}{$p_{0}=10^{15}$} & \multicolumn{2}{c|}{$p_{0}=10^{20}$} \\
\hline$r$ & $k$ & $c_{2}^{\prime}(r)$ & $k$ & $c_{2}^{\prime}(r)$ & $k$ & $c_{2}^{\prime}(r)$ \\
\hline 2 & 0.124 & 3.65 & 0.124 & 3.58 & 0.124 & 3.57 \\
\hline 3 & 0.126 & 2.58 & 0.131 & 2.51 & 0.135 & 2.49 \\
\hline 4 & 0.106 & 2.19 & 0.116 & 2.12 & 0.120 & 2.10 \\
\hline 5 & 0.091 & 1.98 & 0.101 & 1.92 & 0.107 & 1.90 \\
\hline 6 & 0.080 & 1.85 & 0.090 & 1.79 & 0.095 & 1.77 \\
\hline 7 & 0.072 & 1.75 & 0.079 & 1.70 & 0.084 & 1.68 \\
\hline 8 & 0.064 & 1.68 & 0.071 & 1.635 & 0.077 & 1.61 \\
\hline 9 & 0.058 & 1.625 & 0.065 & 1.58 & 0.070 & 1.56 \\
\hline 10 & 0.054 & 1.579 & 0.060 & 1.54 & 0.064 & 1.52 \\
\hline
\end{tabular}

TABLE 7. Values chosen for $k$ and $c_{2}^{\prime}(r)$ to build Table 3 .

Proof of Corollary 2. By Theorem 2, we have our desired result whenever $N<$ $2 p^{\frac{1}{2}+\frac{1}{4 r}}$. Therefore, the only thing we need to prove is that for $p \geq 10^{10}$ and $r \geq 3$, $N<2 p^{\frac{1}{2}+\frac{1}{4 r}}$. Since the induction in the proof of Theorem 2 relied on the upper bound for $N$, we can't use the Burgess inequalities in Theorem 2 to give an upper bound for $N$ in this corollary. However, we can use the Burgess inequalities from Theorem 1 to improve the upper bound for $N$. Indeed, for $p \geq 10^{10}$, we have

$$
\left|S_{\chi}(M, N)\right| \leq 2.6 N^{1-\frac{1}{2}} p^{\frac{3}{16}}(\log p)^{\frac{1}{2}} .
$$

If

$$
N \geq(2.6)^{\frac{2 r}{r-1}} p^{\frac{3}{8}-\frac{1}{8 r}-\frac{3}{8 r(r-1)}} \sqrt{\log p},
$$

then

$$
N^{1-\frac{1}{r}} p^{\frac{r+1}{4 r^{2}}}(\log p)^{\frac{1}{2 r}} \geq 2.6 N^{1-\frac{1}{2}} p^{\frac{3}{16}} \sqrt{\log p} \geq\left|S_{\chi}(M, N)\right| .
$$


Therefore, we may assume that

$$
N \leq(2.6)^{\frac{2 r}{r-1}} p^{\frac{3}{8}-\frac{1}{8 r}-\frac{3}{8 r(r-1)}} \sqrt{\log p} .
$$

Now, all we need to conclude is to show that the right hand side of (60) is less than $2 p^{\frac{1}{2}+\frac{1}{4 r}}$. Using that $p \geq 10^{10}$, we can verify this manually for $r \in\{3,4, \ldots, 21\}$. Now, for $r \geq 22$ we have

$$
N \leq(2.6)^{\frac{2 r}{r-1}} p^{\frac{3}{8}-\frac{1}{8 r}-\frac{3}{8 r(r-1)}} \sqrt{\log p} \leq(2.6)^{\frac{44}{21}} p^{\frac{3}{8}} \sqrt{\log p}<2 p^{\frac{1}{2}} .
$$

The last inequality is true whenever $p \geq 10^{10}$.

Remark 5. Booker and McGown in their proofs have $A$ range through only prime numbers. This idea makes the constants converge quicker. For large enough $p$, it doesn't improve the numbers, but it does for smaller $p$. To save space, we ommited using that technique here, instead focusing on other techniques that made an impact on the "asymptotic" constant. One of the nice ideas not used by McGown or Booker is the idea of using Burgess for smaller $r$ to help out with the larger $r$. This allows the theorems to extend to the whole range when $r \geq 3$.

Remark 6. Theorem A is a little stronger in 21 when the order of the character is bigger. Therefore, one could use that theorem to get better constants for cubic characters, quartic characters and so on.

\section{LEAST $k$-TH POWER NON-RESIDUE}

To prove our results on the least $k$-th power non-residues, we will need the following estimates from [19]:

Lemma 7. Let $B=\lim _{m \rightarrow \infty} \sum_{p \leq m} \frac{1}{p}-\log \log x$, and let $\pi(x)$ be the number of primes up to $x$. Then the following estimates are true:

$$
\begin{gathered}
\log \log x+B-\frac{1}{2 \log ^{2} x}<\sum_{p \leq x} \frac{1}{p} \text { for } x>1, \\
\sum_{p \leq x} \frac{1}{p}<\log \log x+B+\frac{1}{2 \log ^{2} x} \text { for } x \geq 286, \\
\pi(x)<\frac{x}{\log x}\left(1+\frac{3}{2 \log x}\right) \text { for } x>1 .
\end{gathered}
$$

From it we derive the following immediate corollary:

Corollary 3. For real numbers $x$, $y$ satisfying $x>y>1$ and $x \geq 286$, the following estimate is true:

$$
\sum_{y<p \leq x} \frac{1}{p}<\log \log x-\log \log y+\frac{1}{2 \log ^{2} x}+\frac{1}{2 \log ^{2} y} .
$$

Now we are ready to prove the key lemma (a lower bound on a character sum), which is the essence of Vinogradov's trick. 
Lemma 8. Let $x \geq 286$ be a real number, and let $y=x^{\frac{1}{\sqrt{e}}+\delta}$ for some $\delta>0$. Let $\chi$ be a non-principal character $\bmod p$ for some prime $p$. If $\chi(n)=1$ for all $n \leq y$, then

$$
\left|\sum_{n \leq x} \chi(n)\right| \geq x\left(2 \log (\delta \sqrt{e}+1)-\frac{1}{\log ^{2} x}-\frac{1}{\log ^{2} y}-\frac{1}{x}\right) .
$$

Proof. Since $\chi(n)$ is totally multiplicative, $\chi(n)=1$ for all $n \leq y$, and $x<p$, then

$$
\begin{aligned}
\left|\sum_{n \leq x} \chi(n)\right| & =\left|\sum_{\substack{n \leq x \\
\chi(n)=1}} \chi(n)+\sum_{\substack{n \leq x \\
\chi(n) \neq 1}} \chi(n)\right| \\
& =\left|\sum_{n \leq x} 1-\sum_{\substack{n \leq x \\
\chi(n) \neq 1}} 1+\sum_{\substack{n \leq x \\
\chi(n) \neq 1}} \chi(n)\right| \\
& \geq \sum_{n \leq x} 1-2 \sum_{\substack{n \leq x \\
\chi(n) \neq 1}} \chi(n) \\
& \geq \sum_{n \leq x} 1-2 \sum_{\substack{y<q \leq x \\
\chi(q) \neq 1}} \sum_{n \leq \frac{x}{q}} 1,
\end{aligned}
$$

where the sum ranges over $q$ prime. Therefore we have

$$
\left|\sum_{n \leq x} \chi(n)\right| \geq\lfloor x\rfloor-2 \sum_{y<q \leq x}\left\lfloor\frac{x}{q}\right\rfloor \geq x-1-2 x \sum_{y<q \leq x} \frac{1}{q} .
$$

Using Corollary 3 to estimate the sum of the reciprocals of primes we get the desired inequality.

We can now prove Theorem 3. We will use the explicit Burgess inequality proved as Corollary 1 because it works for all $r$.

Proof of Theorem 3. Let $\chi$ be a character $\bmod p$. Then if $n<p$ and $\chi(n) \neq 1, n$ is a $k$-th power non-residue. Let $r$ be an integer. Let $x \geq 286$ be a real number and let $y=x^{\frac{1}{\sqrt{e}}+\delta}=p^{1 / 6}$ for some $\delta>0$. Assume that $\chi(n)=1$ for all $n \leq y$. Now by Corollary 1 and Lemma 8 we have

$$
2.74 x^{1-\frac{1}{r}} p^{\frac{r+1}{4 r^{2}}}(\log p)^{\frac{1}{r}} \geq x\left(2 \log (\delta \sqrt{e}+1)-\frac{1}{\log ^{2} x}-\frac{1}{\log ^{2} y}-\frac{1}{x}\right) .
$$

Now, letting $x=p^{\frac{1}{4}+\frac{1}{2 r}}$ we get

$$
2.74 p^{\frac{\log \log p}{r \log p}-\frac{1}{4 r^{2}}} \geq 2 \log (\delta \sqrt{e}+1)-\frac{1}{\log ^{2} x}-\frac{1}{\log ^{2} y}-\frac{1}{x} .
$$

Picking $r=22$, one finds that $\delta=0.00458 \ldots$ For $p \geq 10^{4732}$, the right hand side of (61) is bigger than the left hand side, showing that $\chi(n)$ is not always 1 for $n \leq y=p^{1 / 6}$, and hence the theorem is true. 
Remark 7. To be able to use Theorem 2 to improve Theorem 3, we would need to calculate what happens for $r>20$ since the restriction $y=p^{1 / 6}$ implies $r>20$. Since we know that $p$ will be large, we can also pick a large $p_{0}$ and then find a good constant for the Burgess inequality when $p$ is very large and $r>20$. After doing all of this work, one could show that Theorem 3 works for $p \geq 10^{3850}$.

\section{ACKNOWLEDGEMENTS}

I would like to dedicate this paper to Paul Bateman and Heini Halberstam. I would like to thank Carl Pomerance for suggesting the problem, his useful comments and his encouragement. I would like to express my gratitude to Andrew Granville, Hugh Montgomery and Robert Vaughan for allowing me to look at unpublished manuscripts that helped me on my work on the Burgess inequality. I would also like to thank Paul Pollack who directed me to McGown's work. Finally, I'd like to thank an anonymous referee that read the paper carefully and suggested many improvements.

\section{REFERENCES}

1. N. C. Ankeny, The least quadratic non residue, Ann. of Math. (2) 55 (1952), 65-72. MR 0045159 (13,538c)

2. Eric Bach, Explicit bounds for primality testing and related problems, Math. Comp. 55 (1990), no. 191, 355-380. MR 1023756 (91m:11096)

3. Andrew R. Booker, Quadratic class numbers and character sums, Math. Comp. 75 (2006), no. 255, 1481-1492 (electronic). MR 2219039 (2008a:11140)

4. A. A. Buhštab, On those numbers in an arithmetic progression all prime factors of which are small in order of magnitude, Doklady Akad. Nauk SSSR (N.S.) 67 (1949), 5-8. MR 0030995 $(11,84 \mathrm{~b})$

5. D. A. Burgess, The distribution of quadratic residues and non-residues, Mathematika 4 (1957), 106-112. MR 0093504 (20 \#28)

6. _ On character sums and primitive roots, Proc. London Math. Soc. (3) 12 (1962), 179-192. MR 0132732 (24 \#A2569)

7. (1963), 253-256. MR 0148628 (26 \#6135)

8. - On character sums and L-series. II, Proc. London Math. Soc. (3) 13 (1963), 524-536. MR 0148626 (26 \#6133)

9. ㄴ The character sum estimate with $r=3$, J. London Math. Soc. (2) 33 (1986), no. 2, 219-226. MR 838632 (87g:11098)

10. H. Davenport and P. Erdös, The distribution of quadratic and higher residues, Publ. Math. Debrecen 2 (1952), 252-265. MR 0055368 (14,1063h)

11. John Friedlander, Primes in arithmetic progressions and related topics, Analytic number theory and Diophantine problems (Stillwater, OK, 1984), Progr. Math., vol. 70, Birkhäuser Boston, Boston, MA, 1987, pp. 125-134. MR 1018373 (90h:11086)

12. G. H. Hardy and E. M. Wright, An introduction to the theory of numbers, Oxford, at the Clarendon Press, 1954, 3rd ed. MR 0067125 (16,673c)

13. Adolf Hildebrand, A note on Burgess' character sum estimate, C. R. Math. Rep. Acad. Sci. Canada 8 (1986), no. 1, 35-37. MR 827113 (87e:11095)

14. Henryk Iwaniec and Emmanuel Kowalski, Analytic number theory, American Mathematical Society Colloquium Publications, vol. 53, American Mathematical Society, Providence, RI, 2004. MR 2061214 (2005h:11005)

15. Kevin J. McGown, Norm-Euclidean cyclic fields of prime degree, Int. J. Number Theory 8 (2012), no. 1, 227-254. MR 2887892

16. Hugh L. Montgomery and Robert C. Vaughan, Multiplicative number theory. I. Classical theory, Cambridge Studies in Advanced Mathematics, vol. 97, Cambridge University Press, Cambridge, 2007. MR 2378655 (2009b:11001) 
17. L. Moser and R. A. MacLeod, The error term for the squarefree integers, Canad. Math. Bull. 9 (1966), 303-306. MR 0200251 (34 \#150)

18. Karl K. Norton, Numbers with small prime factors, and the least kth power non-residue, Memoirs of the American Mathematical Society, No. 106, American Mathematical Society, Providence, R.I., 1971. MR 0286739 (44 \#3948)

19. J. Barkley Rosser and Lowell Schoenfeld, Approximate formulas for some functions of prime numbers, Illinois J. Math. 6 (1962), 64-94. MR 0137689 (25 \#1139)

20. Enrique Treviño, Numerically explicit estimates for character sums, 2011, Thesis (Ph.D.)Dartmouth College.

21. Enrique Treviño, The least $k$-th power non-residue, J. Number Theory 149 (2015), 201-224. MR 3296008

22. Ivan Matveevič Vinogradov, Selected works, Springer-Verlag, Berlin, 1985, With a biography by K. K. Mardzhanishvili, Translated from the Russian by Naidu Psv [P. S. V. Naidu], Translation edited by Yu. A. Bakhturin. MR 807530 (87a:01042)

23. André Weil, On some exponential sums, Proc. Nat. Acad. Sci. U. S. A. 34 (1948), 204-207. MR 0027006 (10,234e)

24. W. Yuan, Estimation and application of character sums, Shuxue Jinzhan 7 (1964), 78-83.

Department of Mathematics and Computer Science, Lake Forest College, Lake ForEST, ILlinois 60045

E-mail address: trevino@lakeforest.edu 